\title{
INJECTOR DESIGN FOR THE 4GLS HIGH AVERAGE CURRENT LOOP
}

\author{
J.W. McKenzie*, B.L. Militsyn, STFC Daresbury Laboratory, Warrington, WA4 4AD, UK \\ A.S. Terekhov, ISP, Novosibirsk, 630090, Russian Federation
}

\begin{abstract}
The proposed 4th Generation Light Source (4GLS) consists of three electron branches. We present the design of the injector for the High Average Current Loop which feeds spontaneous light sources and a Vacuum UltraViolet FEL. The injector aims to provide 77 pC bunches at a repetition rate of up to $1.3 \mathrm{GHz}$ which corresponds to an average current of $100 \mathrm{~mA}$. It consists of a $500 \mathrm{kV}$ GaAs based DC photocathode electron gun equipped with a photocathode preparation facility, followed by a normalconducting buncher cavity and a $10 \mathrm{MeV}$ superconducting RF booster. Simulations are presented which show the injector provides a beam with a normalised rms transverse emittance of less than $3 \pi \cdot \mathrm{mm} \cdot \mathrm{mrad}$ and a bunch length of about 2 ps.
\end{abstract}

\section{INTRODUCTION}

The 4GLS facility which is under development at Daresbury Laboratory consists of three inter-related accelerator branches driving a variety of spontaneous light sources and free-electron lasers (FELs) operating in the Infra-Red (IR), Vacuum Ultra-Violet (VUV) and soft $\mathrm{X}$-ray regimes. A conceptual design report has been published [1] and work is currently under way for a technical design report due in 2008. Three separate injectors are required. A grid-modulated thermionic gun will drive the 25-60 MeV IR-FEL branch, a 1.5 cell normal conducting RF gun will drive the $750 \mathrm{MeV}$ XUVFEL branch and a DC photo-injector will drive the High Average Current Loop (HACL). This paper discusses the design and simulations of the HACL injector.

The HACL branch of 4GLS is a single pass energy recovery system which aims to deliver an electron beam of $550 \mathrm{MeV}$ with an average current of up to $100 \mathrm{~mA}$ through five insertion device straights, the last of which contains a VUV-FEL. The beam is accelerated by a superconducting linac which is shared with the beam of the XUV-FEL branch operating at a different bunch charge and frequency. Details of the beam dynamics of the entire system are given elsewhere [2].

The HACL injector needs to provide a beam of $10 \mathrm{MeV}$ and of high quality for the insertion devices. These require a normalised emittance of less than $2 \pi \cdot \mathrm{mm} \cdot \mathrm{mrad}$ and an uncorrelated energy spread of less than $0.1 \%$. The injector also needs to provide a bunch length of less than $3 \mathrm{ps}$ in order for the compression system to achieve the required $100 \mathrm{fs}$ at the entrance of VUV-FEL whilst using sextupoles for linearization [2]. The average current of $100 \mathrm{~mA}$ can be reached by filling every $1.3 \mathrm{GHz} \mathrm{RF}$ bucket with a bunch of charge $77 \mathrm{pC}$.

*j.w.mckenzie@dl.ac.uk

\section{INJECTOR DESIGN}

The basis of the injector is a GaAs DC photocathode gun which is followed by a solenoid and a single-cell buncher cavity based on the Cornell design [3]. The buncher cavity is followed by a second solenoid and two $5 \mathrm{MeV}$ superconducting booster sections [4]. These sections each contain five two-cell cavities which have independently adjustable phase and gradients. Fig. 1 shows a schematic layout of the injector.

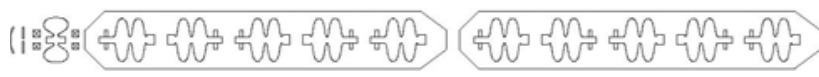

Figure 1: Injector layout.

\section{Electron Gun}

A DC photocathode gun will operate at $500 \mathrm{kV}$ with a negative electron affinity GaAs photocathode. This will be back-illuminated by a laser providing a $1.3 \mathrm{GHz}$ train of pulses synchronised to the linac RF. Back-illuminating the photocathode reduces scattering of light producing unwanted electrons outside the main beam by means of an anti-scattering coating. It also allows for lower laser power to be used thus reducing heating of the cathode.

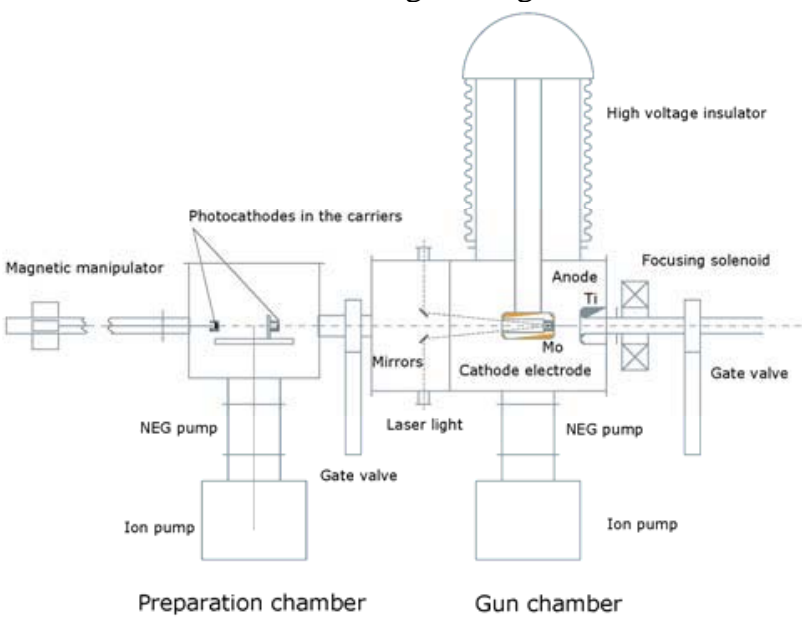

Figure 2: Schematic of the electron gun.

The main limiting factor of the gun performance is the operational lifetime of the photocathode which is restricted by the back ion stream. Residual gas becomes ionized by the primary electron beam and then accelerated towards the photocathode surface, degrading it, resulting in a decrease in the quantum efficiency $\left(\mathrm{Q}_{\mathrm{e}}\right)$. An extremely high level of vacuum is required to limit this damage. The photocathode lifetime is expected to be around 24 hours and so to minimise downtime between cathode changes, the gun is equipped with a multiple chamber cathode preparation facility as shown in Figs. 2 
and 3. The photocathode will be installed in the gun using a load-lock system from a preparation chamber. This chamber can hold up to 5 photocathodes and includes facilities for heat treatment and activation of the photocathodes with $\mathrm{Cs}$ and $\mathrm{O}_{2}$ or $\mathrm{NF}_{3}$. In addition it is proposed to have a dedicated hydrogen cleaning chamber to repair degraded photocathodes. Before loading into the gun system, new photocathodes will be chemically cleaned in a nitrogen filled glove box.

It is planned to have a titanium anode and a molybdenum cathode as these electrode materials have been observed to exhibit very low field emission [5]. A cathode electrode angle of $10^{\circ}$ was chosen as this ensures a constant transverse beam size throughout the gun up to the first solenoid. The GaAs wafer is planned to be $12 \mathrm{~mm}$ in diameter to allow a laser spot of diameter $6 \mathrm{~mm}$ to be operated off-axis, if required, to reduce cathode degradation due to ion back-bombardment which is at a maximum on-axis. The laser spot size was chosen as a compromise between a small spot for low emittance and a large spot to improve cathode lifetime by spreading out damage from ion back-bombardment [6].

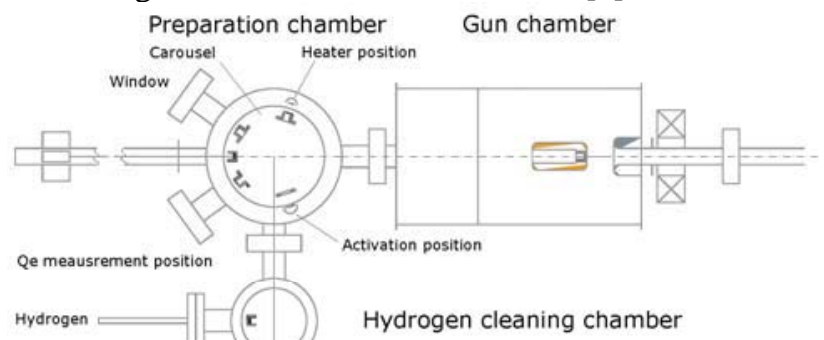

Figure 3: Schematic showing the different chambers.

\section{Photocathode Development}

Extensive work is being carried out to develop a photocathode to meet the requirements of delivering $100 \mathrm{~mA}$ with a high quality. The photocathode should provide a short time response approaching $20 \mathrm{ps.}$ However, the longitudinal beam profile from a bulk GaAs photocathode possesses an undesirable long tail due to the long absorption and diffusion lengths in the semiconductor [7]. A profile approaching a Gaussian can be achieved by limiting the active layer thickness to as low as $200 \mathrm{~nm}$ [8]. Assuming photon energy is equal to the semiconductor band gap, an active layer this thin does not allow complete absorption of incident light. This reduces the $Q_{e}$ to only a few percent compared to the $40 \%$ that can be achieved with a bulk material. Therefore to reach the desired $100 \mathrm{~mA}$ the photocathode will have to be illuminated with laser power approaching $50 \mathrm{~W}$. This level of power heats the GaAs to a temperature at which the active layer begins to decay. Very efficient photocathode cooling is required and this has led to the development of new structures on durable substrates since conventional photocathodes are too fragile to be pressed on to the mounting position with sufficient pressure to provide low thermal resistance. Both reflection-mode photocathodes bonded to molybdenum and silicon and transmission-mode (for back-illumination) on sapphire are currently under development.

\section{INJECTOR MODELLING}

Beam dynamic simulations from the cathode up to the exit of the second SRF booster were performed using ASTRA [9]. On-axis field distributions in the electron gun were calculated with POISSON and used as an input into ASTRA. The beam on the cathode was modelled transversely with a $6 \mathrm{~mm}$ diameter "top-hat" profile and longitudinally with a 20 ps rms Gaussian. These lengths were chosen to encompass all possibilities as the laser profile and cathode dimensions have not been finalised. An initial energy spread of $200 \mathrm{meV}$ was included that gives a thermal emittance of $0.94 \pi \cdot \mathrm{mm} \cdot \mathrm{mrad}$.

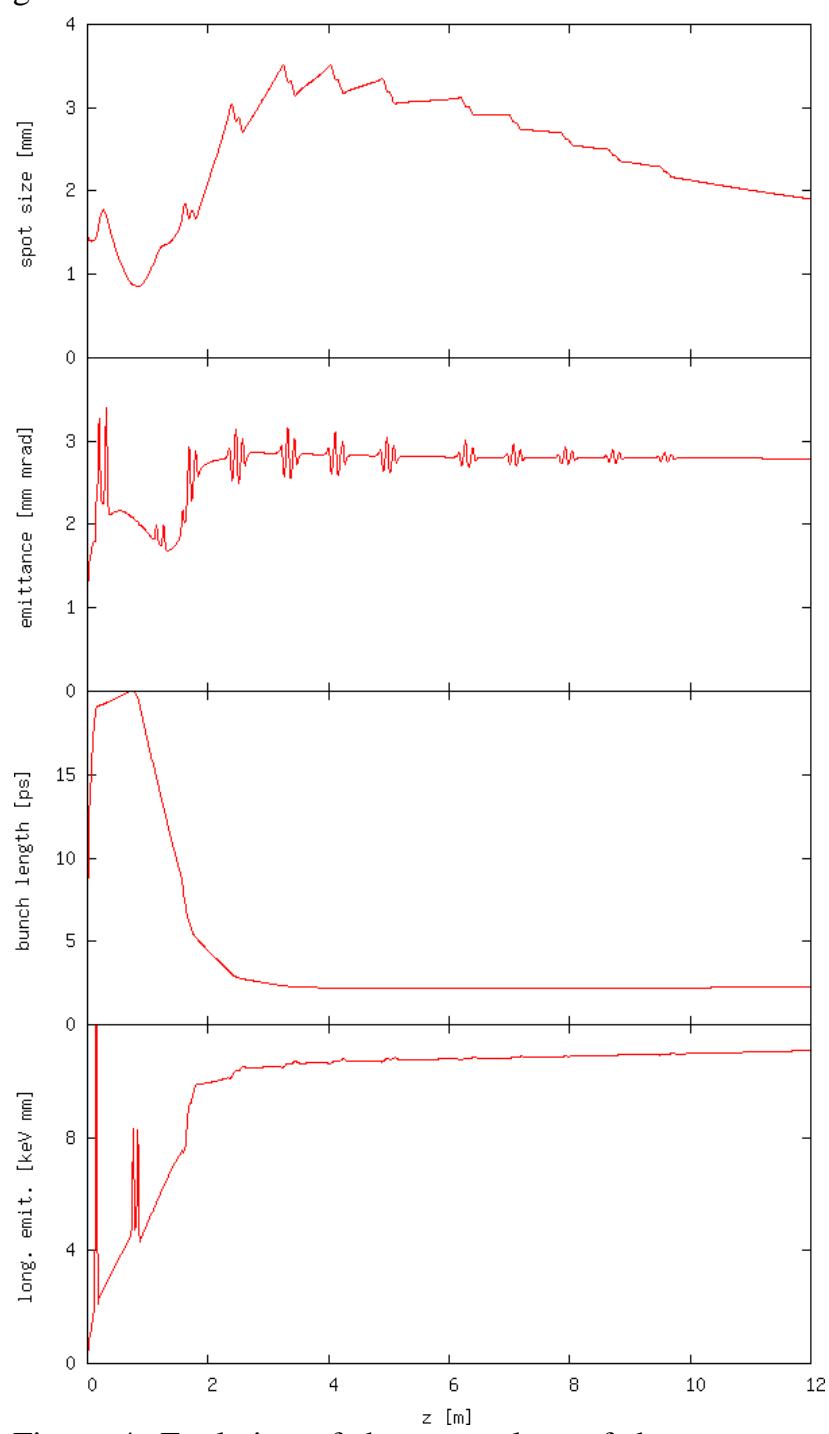

Figure 4: Evolution of the rms values of the transverse beam size, normalised transverse emittance, bunch length, and longitudinal emittance along the injection line. 
The injector layout was designed to minimise the length whilst leaving enough room for diagnostic placement. The first solenoid field strength was set to produce a minimum of spot size to pass through the buncher aperture and maintain a transverse emittance of $2 \pi \cdot \mathrm{mm} \cdot \mathrm{mrad}$ up to the first booster cavity. The main problem with the optimisation is simultaneously achieving a small emittance along with a bunch length as low as 2 ps. It was found that it was not possible to just use the buncher cavity to reduce the bunch length from 20 ps to 2 ps as this causes large emittance blow-up at the entrance to the booster. The buncher has been set to reduce the bunch length down to $6 \mathrm{ps}$ at the booster entrance however this still causes an emittance growth of $\sim 1 \pi \cdot \mathrm{mm} \cdot \mathrm{mrad}$. The first superconducting cavity has been set to a negative phase to provide the final bunching from 6 ps to 2 ps.

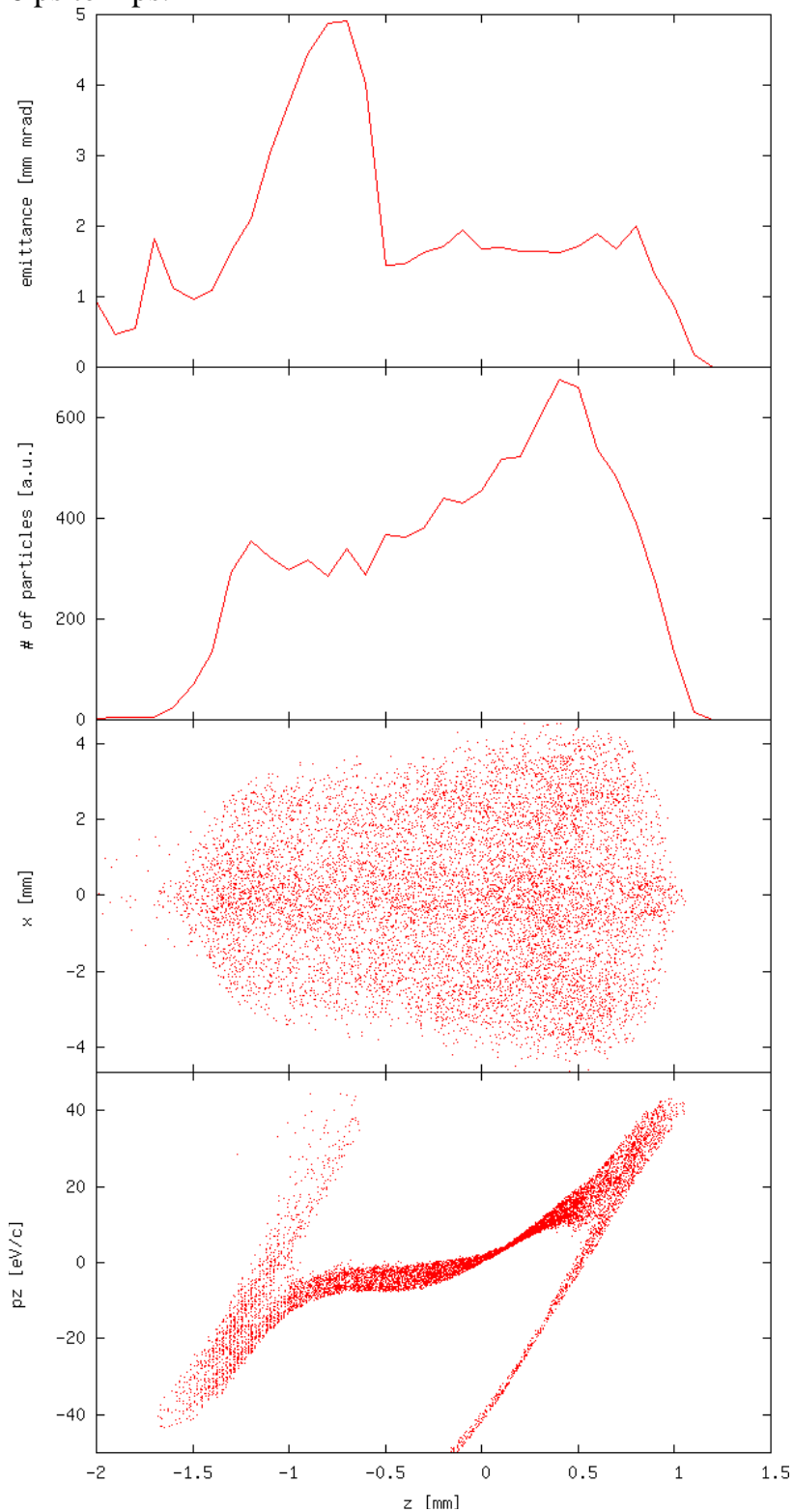

Figure 5: Slice emittance, particle distribution, bunch shape and longitudinal phase space at the injector exit.
Table 1: Final beam parameters after $12 \mathrm{~m}$.

\begin{tabular}{|l|l|}
\hline Transverse emittance & $2.8 \pi \cdot \mathrm{mm} \cdot \mathrm{mrad}$ \\
\hline Bunch length & $2.2 \mathrm{ps}$ \\
\hline Energy spread & $0.2 \%$ \\
\hline Longitudinal emittance & $11 \pi \cdot \mathrm{keV} \cdot \mathrm{mm}$ \\
\hline
\end{tabular}

With the current values of solenoid and buncher field strength, adjusting the phase and gradient beyond the $2^{\text {nd }}$ cavity produces no improvement in either emittance or bunch length. As such the parameters of these cavities have simply been adjusted to allow the final energy of $10.5 \mathrm{MeV}$ to be reached. Fig. 4 shows the evolution of the beam parameters along the injection line and Table 1 summarises them at the exit of the injector. As can be seen, the emittance is larger than the required $2 \pi \cdot \mathrm{mm} \cdot \mathrm{mrad}$ however as Fig. 5 shows, the slice emittance remains below $2 \pi \cdot \mathrm{mm} \cdot \mathrm{mrad}$ for the majority of the bunch apart from a chirp at the tail.

\section{SUMMARY}

The ASTRA simulations show that the current injector design is capable of achieving the required performance for 4GLS. Further work will be carried out on the design and fabrication of cooled photocathodes and measurements taken of the initial energy distribution of the emitted electrons. Further simulations incorporating these results and a full 3D model of the electron gun will then be performed.

\section{REFERENCES}

[1] “4GLS Conceptual Design Report”, CCLRC Daresbury Laboratory (2006), available at www.4gls.ac.uk.

[2] P.H. Williams et al, "Electron Beam Dynamics in 4GLS", these proceedings.

[3] V. Veshcherevich and S. Belomestnykh, "Buncher Cavity for ERL”, PAC'03.

[4] V. Medjidzade et al, "Design of the CW Cornell ERL Injector Cryomodule”, PAC’05

[5] F. Furuta et al, "Reduction of field emission dark current for high-field gradient electron gun by using a molybdenum cathode and titanium anode", NIM A, 538 (2005) 33-44.

[6] J. Grames et al, "Measurements of Photocathode Operational Lifetime at Beam Currents up to $10 \mathrm{~mA}$ using an Improved DC High Voltage GaAs Photogun", SPIN'06

[7] P. Hartmann et al, "A diffusion model for picosecond electron bunches from negative electron affinity GaAs photocathodes”, J. Appl. Phys. 86 (1999) 2245-2249.

[8] K. Aulenbacher et al, "Pulse response of thin III/V semiconductor photocathodes”, J. Appl. Phys. 92 (2002) 7536-7543.

[9] K. Flöttmann, ASTRA homepage, http://www.desy.de/ mpyflo/ 